\title{
ON BAHADUR'S REPRESENTATION OF SAMPLE QUANTILES
}

\author{
By J. KIEFER ${ }^{1}$ \\ Cornèll University
}

1. Introduction and summary. Let $X_{1}, X_{2}, \cdots$ be independent and identically distributed real random variables with common df $F$. Suppose that $0<p<1$, that $F\left(\xi_{p}\right)=p$, that $F$ is twice differentiable in a neighborhood of $p$, and that $F^{\prime \prime}$ is bounded in that neighborhood and $F^{\prime}\left(\xi_{p}\right)>0$. Let $S_{n}$ be the sample df based on $\left(X_{1}, \cdots, X_{n}\right)$; i.e., $n S_{n}(x)=$ number of $X_{i} \leqq x, 1 \leqq i \leqq n$. Let $Y_{p, n}$ be a sample $p$-quantile based on $\left(X_{1}, \cdots X_{n}\right)$; i.e., $S_{n}\left(Y_{p, n}-\right) \leqq p \leqq$ $S_{n}\left(Y_{p, n}\right)$; if $n p$ is an integer, so that $Y_{p, n}$ is not unique, it will be seen that any measurable definition can be used in the sequel, and for the sake of definiteness we shall take the smallest possible value. We shall write $\sigma_{p}=[p(1-p)]^{\frac{1}{2}}$.

Let'

$$
R_{n}(p)=Y_{p, n}-\xi_{p}+\left[S_{n}\left(\xi_{p}\right)-p\right] / F^{\prime}\left(\xi_{p}\right) .
$$

Bahadur (1966) initiated the study of $R_{n}(p)$ and showed that

$$
R_{n}(p)=O\left(n^{-3 / 4}(\log n)^{\frac{1}{2}}(\log \log n)^{\frac{1}{4}}\right)
$$

wp 1 as $n \rightarrow \infty$. He also raised the question of finding the exact order of $R_{n}(p)$. In the present paper we answer this by proving

Theorem 1. For either choice of sign,

(1.3) $\lim \sup _{n \rightarrow \infty} \pm F^{\prime}\left(\xi_{p}\right) R_{n}(p) /\left[2^{5 / 4} 3^{-3 / 4} \sigma_{p}^{\frac{1}{2}} n^{-3 / 4}(\log \log n)^{3 / 4}\right]=1$

\section{wp 1 .}

Later in this section we shall sketch the rationale behind this result, whose proof will occupy most of the paper.

Let $\tau$ be any positive number. For $t \varepsilon[-\tau, \tau]$, we write

$$
\begin{gathered}
U_{n}(t)=n^{3 / 4} F^{\prime}\left(\xi_{p+n^{-\frac{1}{2}} t} t\right) R_{n}\left(p+n^{-\frac{1}{2}} t\right), \\
U_{n} \equiv U_{n}(0)=n^{3 / 4} F^{\prime}\left(\xi_{p}\right) R_{n}(p),
\end{gathered}
$$

which by assumption on $F$ make sense for $n$ large. Also write

$$
K_{n}=n^{\frac{1}{2}}\left(p-S_{n}\left(\xi_{p}\right)\right) \text {. }
$$

As the discussion later in this section shows, it is trivial that, for each $b>0$, uniformly in $b^{-1}<\left|k_{n}\right|<b$, the conditional law of $U_{n} /\left|k_{n}\right|^{\frac{1}{2}}$, given $K_{n}=k_{n}$, is asymptotically $N(0,1)$ as $n \rightarrow \infty$. Since $K_{n} / \sigma_{p}$ is also asymptotically $N(0,1)$, it is obvious that

$$
\lim _{n \rightarrow \infty} P\left\{U_{n} \leqq u\right\}=2 \sigma_{p}^{-1} \int_{0}^{\infty} \Phi\left(k^{-\frac{1}{2}} u\right) \phi\left(k / \sigma_{p}\right) d k,
$$

where $\Phi$ and $\phi$ are the standard $N(0,1)$ df and density. More generally, we shall

Received 22 December 1966.

${ }_{1}^{1}$ Research supported by the Office of Naval Research under Contract No. NONR 401 (50). Reproduction in whole or in part is permitted for any purpose of the United States Government. 
show in Section 5 that, under the same conditioning, and uniformly for $k_{n}$ in a set of probability arbitrarily close to 1 according to the law of $K_{n}$ for each large $n$, for each finite collection $t_{1}, t_{2}, \cdots, t_{m}$ of values in $[-\tau, \tau]$, the asymptotic joint conditional df of $\left\{U_{n}\left(\left|k_{n}\right| t_{1}\right) /\left|k_{n}\right|^{\frac{1}{2}}, \cdots, U_{n}\left(\left|k_{n}\right| t_{m}\right) /\left|k_{n}\right|^{\frac{1}{2}}\right\}$ is the same as the df of $\left\{J\left(t_{1}\right), \cdots, J\left(t_{m}\right)\right\}$, where $\{J(t),-\infty<t<\infty\}$ is a separable stationary Gaussian process with mean 0 and convariance function

$$
E\left\{J\left(t_{1}\right) J\left(t_{2}\right)\right\}=\max \left(1-\left|t_{1}-t_{2}\right|, 0\right) .
$$

(The definition of $U_{n}\left(\left|k_{n}\right| t\right) /\left|k_{n}\right|^{\frac{1}{2}}$ can be made arbitrarily when $k_{n}=0$, since $P\left\{K_{n}=0\right\} \rightarrow 0$. $)$ Let $G$ be $N\left(0, \sigma_{p}{ }^{2}\right)$ and independent of the $J$-process. Let $J^{*}(t)=|G|^{\frac{1}{2}} J(t /|G|)$. Our discussion above indicates a result we shall prove in Section 5.

Theorem 2. As $n \rightarrow \infty$ the process $\left\{U_{n}(t),|t| \leqq \tau\right\}$ approaches in law the process $\left\{J^{*}(t),|t| \leqq \tau\right\}$, whose sample paths are Lip $\alpha$ for all $\alpha<\frac{1}{2}$, wp 1., and $\sup _{|t| \leqq \tau} \pm U_{n}(t)$ approaches $\sup _{|t| \leqq \tau} \pm J^{*}(t)$ in law.

We shall also prove, in Section 5:

THeOREM 3. For either choice of sign, (1.3) holds if $\pm n^{3 / 4} F^{\prime}\left(\xi_{p}\right) R_{n}(p)$ is replaced there by $\sup _{|t| \leqq \tau}\left[ \pm U_{n}(t)\right]$.

Actually, this can be strengthened by letting $\tau \rightarrow \infty$ slowly with $n$, as in (5.4), but this is of limited interest. (See also Remark 2 of Section 6.)

Section 6 contains other remarks on related results in the domain of Bahadur's considerations, including (Theorem 4 ) the observation that his technique yields the strong form of the law of the iterated logarithm for order statistics, and not just the classical form as exhibited in Equation (4) of [1], and also (Theorem 5) the validity for sample quantiles of analogues of Strassen's results [11] for the cumulative sums $n S_{n}(p)$. Section 2 collects most of the elementary probabilistic estimates which are needed in the proof of Theorem 1, the lower- and upper-class parts of which are contained in Sections 3 and 4.

Before proceeding further, we reduce our considerations by the following:

LEMma 1. If Theorem 1, 2, and 3 are valid when $F$ is the uniform df on $[0,1]$, then they are valid for general $F$ satisfying the assumptions stated at the outset of this section.

Proof. For $F$ satisfying the assumptions, define independent uniformly distributed random variables $X_{1}{ }^{*}, X_{2}{ }^{*}, \cdots$ as follows: Let $H_{1}, H_{2}, \cdots$ be uniform on $[0,1]$ and independent of each other and of the original $X_{i}$. Let $X_{i}{ }^{*}=$ $F\left(X_{i-}\right)\left(1-U_{i}\right)+F\left(X_{i}\right) U_{i}$. (If $F$ is continuous, $X_{i}^{*}=F\left(X_{i}\right)$.) Then the $X_{i}^{*}$ have the desired joint uniform distribution. Moreover, if $B$ is the hypothesized neighborhood of $\xi_{p}$ in which $F$ is twice differentiable, and if $S_{n}{ }^{*}$ and $Y_{p, n}^{*}$ are the sample df and sample $p$-quantile based on $\left(X_{1}{ }^{*}, \cdots, X_{n}{ }^{*}\right)$, then Taylor's Theorem with remainder yields for the corresponding $R_{n}{ }^{*}(p)$, as long as $Y_{p, n} \varepsilon B$,

$$
\begin{aligned}
R_{n}{ }^{*}(p) & =Y_{p, n}^{*}-p+S_{n}{ }^{*}(p)-p \\
& =F\left(Y_{p, n}\right)-p+S_{n}\left(\xi_{p}\right)-p \\
& =F^{\prime}\left(\xi_{p}\right)\left(Y_{p, n}-\xi_{p}\right)+F^{\prime \prime}\left(\xi^{\prime}\right)\left(Y_{p, n}-\xi_{p}\right)^{2} / 2+S_{n}\left(\xi_{p}\right)-p \\
& =F^{\prime}\left(\xi_{p}\right) R_{n}(p)+F^{\prime \prime}\left(\xi^{\prime}\right)\left(Y_{p, n}-\xi_{p}\right)^{2} / 2 F^{\prime}\left(\xi_{p}\right),
\end{aligned}
$$


where $\xi^{\prime}$ is a chance value in $B$. Since $Y_{p, n} \varepsilon B$ wp 1 for all large $n$ and since $n^{\frac{1}{2}}\left(Y_{p, n}-\xi_{p}\right)$ in fact obeys a law of the iterated logarithm, we obtain

$$
n^{3 / 4}\left[R_{n}{ }^{*}(p)-F^{\prime}\left(\xi_{p}\right) R_{n}(p)\right] \rightarrow 0 \text { wp } 1 .
$$

This yields the desired result regarding Theorem 1, and the results for Theorems 2 and 3 are obtained similarly; one uses the additional fact that $n^{3 / 4} \sup _{|t| \leqq \tau n-1 / 2}$ $\left(Y_{p+t, n}-\xi_{p+t}\right)^{2} \rightarrow 0 \mathrm{wp} \mathrm{1}$, which is an easy consequence of the law of the iterated logarithm for the sample df (see [2]), or can be proved by the techniques of Section 5.

THROUGHOUT THIS PAPER WE HEREAFTER ASSUME F TO BE THE UNIFORM DF ON [0, 1], UNLESS EXPLICITLY STATED TO THE CONTRARY (POSSIBLY BY EXHIBITING THE SYMBOL $F$ ).

We now introduce some further notation. The complement of an event $A$ will be denoted $\bar{A}$. In the proof of Theorem $1, \gamma$ will be a value $>1$ (large in Section 3 , near 1 in Section 4). For each positive integer $r$, we denote by $n_{r}$ the greatest integer $\leqq \gamma^{r}$. Whenever we write $\sum_{r}$ or $\sum_{n}$ it will be understood that the summation (to $\infty$ ) begins at a large enough value that expressions like log $\log n_{r}$ which appear in the summand are real; a similar remark applies to other expressions. All "orders" refer to behavior as $n \rightarrow \infty$ or $r \rightarrow \infty$. We abbreviate "infinitely often" by "i.o." and "almost all $n$ " (i.e., all natural numbers $n$ except for a finite number) by "a.a. $n$ ". We define

$$
\begin{aligned}
& q_{n}=2^{5 / 4} 3^{-3 / 4} \sigma_{p}^{\frac{1}{2}} n^{-3 / 4}(\log \log n)^{3 / 4}, \\
& h_{n}=2^{\frac{1}{2}} 3^{-\frac{1}{2}} \sigma_{p} n^{-\frac{1}{2}}(\log \log n)^{\frac{1}{2}}
\end{aligned}
$$

and

$$
\begin{aligned}
T_{n}(x) & =S_{n}(x+p)-(x+p)+n^{-\frac{1}{2}} K_{n}, \\
T_{n}{ }^{+}(x) & =\sup _{0 \leqq v \leqq x} T_{n}(v), \\
T_{n}{ }^{-}(x) & =-\inf _{0 \leqq v \leqq x} T_{n}(v), \\
T_{n}{ }^{*}(x) & =\sup _{0 \leqq v \leqq x}\left|T_{n}(v)\right| .
\end{aligned}
$$

Note that $T_{n}(0)=0 \mathrm{wp} 1$.

We now rephrase the statement of Theorem 1 in a form that will make it simpler to explain. Suppose for the moment that the event $K_{n}=k_{n}>0$ occurs. It is then clear that, $F$ being uniform, if $d_{n}>0$, then

$$
\begin{gathered}
R_{n}(p)>d_{n} \Leftrightarrow T_{n}\left(n^{-\frac{1}{2}} k_{n}+d_{n}\right)<-d_{n}, \\
R_{n}(p)<-d_{n} \Leftrightarrow T_{n}\left(n^{-\frac{1}{2}} k_{n}-d_{n}\right) \geqq d_{n},
\end{gathered}
$$

except for a set of probability zero (where $T_{n}$ has a jump at $n^{-\frac{1}{2}} k_{n}-d_{n}$ ) in the second case. (Of course, (1.12) is also valid when $d_{n} \leqq 0$ ). The event of this second line of (1.12) is illustrated in Figure 1 in the case $d_{n}<n^{-\frac{1}{2}} k_{n}$. For the case $K_{n}=k_{n} \leqq 0$, there are two analogous events, again given by (1.12). Referring to the second line of (1.12) with $d_{n}=\lambda q_{n}$, write

$$
A_{n}(\lambda)=\left\{K_{n}>0 \text { and } T_{n}\left(n^{-\frac{1}{2}} K_{n}-\lambda q_{n}\right) \geqq \lambda q_{n}\right\} .
$$




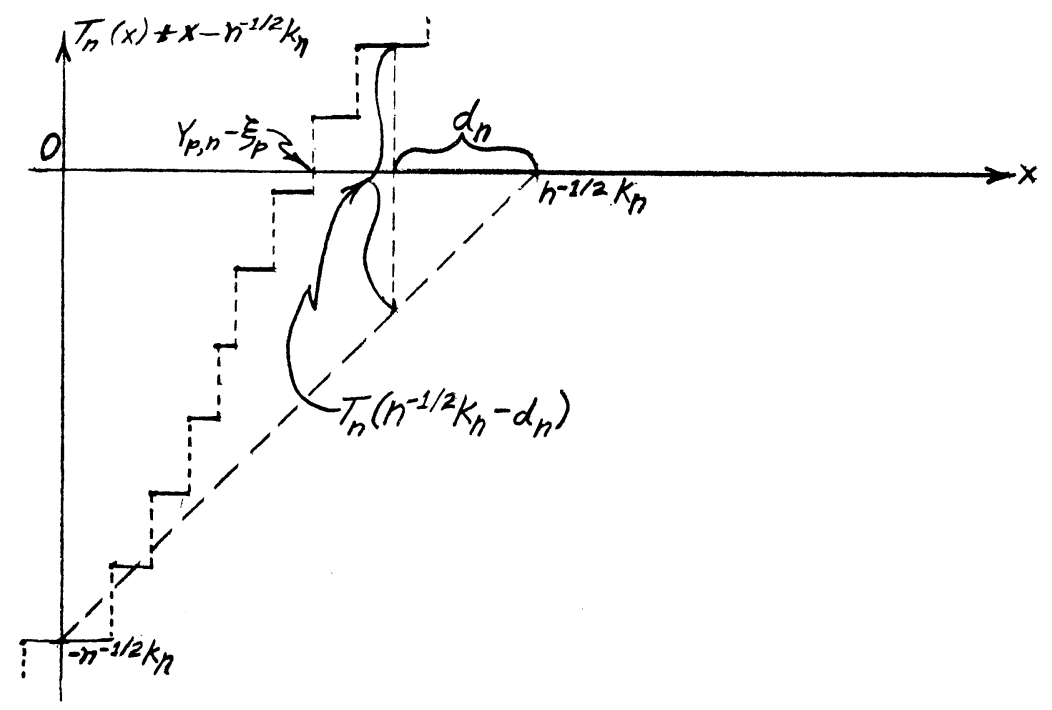

Fig. 1

Suppose we proved that

$$
\begin{aligned}
P\left\{A_{n}(\lambda) \text { i.o. }\right\} & =1 \text { if } \lambda<1, \\
& =0 \text { if } \lambda>1,
\end{aligned}
$$

and also proved the three analogous results for the first case of (1.12) and the two cases of $K_{n} \leqq 0$. This would then clearly prove Theorem 1 . The cases when $K_{n} \leqq 0$ can be obtained by invoking the cases when $K_{n}>0$ for the $(1-p)$ tile of the random variables $1-X_{i}$ and the fact that for $\lambda>0$ the event $\left\{K_{n}=0\right.$, $T_{n}\left( \pm \lambda q_{n}\right) \pm \lambda q_{n}=0$ i.o. $\}$ is easily seen to have probability zero by using the Borel-Cantelli Lemma. Sections 2, 3, and 4 are devoted mainly to proving (1.14). Any modifications which are needed to prove the analogue of (1.14) for the other case of (1.12) are indicated, thus yielding Theorem 1.

As usual, for $N$ a positive integer and $0<\pi<1$, we denote the binomial df by

$$
B(z, N, \pi)=\sum_{j \leqq z}\left(\begin{array}{c}
N \\
j
\end{array}\right) \pi^{j}(1-\pi)^{N-j},
$$

and we also write

$$
B^{*}(\Delta, N, \pi)=B(\Delta+N \pi, N, \pi) .
$$

We shall frequently use the symbols $\Delta, N$, and $\pi$ to refer to quantities in the context (1.16). If $N=0, B$ and $B^{*}$ assign probability one to the value zero.

We now indicate briefly the idea behind the statement (1.14). Fix $\lambda>0$. Then $n^{-\frac{1}{2}} k_{n}>\lambda q_{n}$ clearly implies

$$
\begin{aligned}
& P\left\{A_{n}(\lambda) \mid K_{n}=k_{n}\right\} \\
& \quad=1-B^{*}\left(\lambda n q_{n}-, n\left(1-p+n^{-\frac{1}{2}} k_{n}\right),\left(n^{-\frac{1}{2}} k_{n}-\lambda q_{n}\right) /(1-p)\right) .
\end{aligned}
$$


If $b^{-1}<k_{n}<b$, the Central Limit Theorem is applicable with $\lambda q_{n}$ replaced by $n^{-3 / 4} u$ and (with the corresponding result for $K_{n}<0$ ) yields (1.6). In fact, if $k_{n}=b n^{\frac{1}{2}} h_{n}$ with $b>0$, which normalization turns out to yield the crucial range of values of $K_{n}$, Lemma 2 of Section 2 implies that the normal approximation applies both to (1.17) and to the df of $K_{n}$ at $k_{n}$, and yields

$$
\begin{aligned}
\log P\left\{A_{n}(\lambda) \mid K_{n}=b n^{\frac{1}{2}} h_{n}\right\} & \sim \log \left[1-\Phi\left(\lambda n q_{n} /\left(b n h_{n}\right)^{\frac{1}{2}}\right)\right] \\
& \sim-\frac{1}{2}\left[\left(4 \lambda^{2} / 3 b\right) / \log \log n\right], \\
\log P\left\{K_{n}>b n^{\frac{1}{2}} h_{n}\right\} & \sim \log \left[1-\Phi\left(b n^{\frac{1}{2}} h_{n} / \sigma_{p}\right)\right] \\
& \sim-\frac{1}{2}\left[\left(2 b^{2} / 3\right) \log \log n\right] .
\end{aligned}
$$

Since $\min _{b>0}\left[2 b^{2} / 3+4 \lambda^{2} / 3 b\right]=2 \lambda^{\frac{4}{3}}$, attained at $b=\lambda^{\frac{2}{3}}$, it is not hard to show from (1.18) that

$$
\log P\left\{A_{n}(\lambda)\right\} \sim-\lambda^{4} \log \log n,
$$

so that, whatever $\lambda>1$,

$$
\begin{aligned}
\sum_{r} P\left\{A_{n_{r}}(\lambda)\right\} & =\infty \text { if } \lambda<1, \\
& <\infty \text { if } \lambda>1 .
\end{aligned}
$$

This suggests that (1.14) can be attacked by the classical approach of using the Borel-Cantelli Lemmas for the event $\left\{A_{n_{r}}(\boldsymbol{\lambda})\right.$ i.o. $\}$ when $\lambda>1$ and for a corresponding event in terms of a sequence of independent blocks of observations (from $n_{r}+1$ to $n_{r+1}$ ) when $\lambda<1$, and by then showing that nothing much different happens for intermediate values of $n$.

It is this last aspect of the proof which requires some delicacy and slightly different techniques from those employed in the classical case [5], [6] or in the proof of the sample df law [2], [7]. For example, a step analogous to that of the usual approach (as in [6]) in the case of the upper class would be to show that, if $\lambda>1$, then $\gamma$ and $\lambda^{\prime}>1$ and $\epsilon>0$ can be chosen so that, for all large $r$ and $n_{r}<n \leqq n_{r+1}$,

$$
P\left\{A_{n_{r+1}}\left(\lambda^{\prime}\right) \mid A_{n}(\boldsymbol{\lambda}) \bigcap_{n_{r}<j<n} \overline{A_{j}(\lambda)}\right\}>\epsilon .
$$

But the intuitively obvious (1.21) seems difficult to establish in view of the complexity of the condition of (1.21) compared with the analogue in the case of partial sums or the sample df. Thus, we forego (1.21) and, roughly speaking, prove instead the superficially stronger relation

$$
P\left\{\bigcup_{n_{r}<n<n_{r+1}} \overline{A_{n}(\lambda)} \mid \overline{A_{n_{r}}\left(\lambda^{\prime}\right)}\right\}>1-\epsilon_{r},
$$

where $\sum_{r} \epsilon_{r}<\infty$. Such relations rely heavily on the persistence in $n$ and in $x$ of the sample df deviations $T_{n}(x)$, as made precise in some of the lemmas of Section 2.

2. Preliminary lemmas. Because the last argument of $B^{*}$ in (1.17) is customarily small in our considerations, we shall often require the Central Limit Theorem for small tail probabilities in the context of (1.16) when $N \rightarrow \infty$, 
$\pi \rightarrow 0, N \pi \rightarrow \infty$, and $\Delta^{2} / N \pi \rightarrow \infty$ at a slow enough rate:

Lemma 2. If $\Delta_{N}>0, \pi_{N} \rightarrow 0, N \pi_{N} \rightarrow \infty,{\Delta_{N}}^{2} / N \pi_{N} \rightarrow \infty$, and $\Delta_{N}{ }^{3} / N^{2}{\pi_{N}}^{2} \rightarrow 0$, then, for each $\zeta>0$, as $N \rightarrow \infty$,

$$
\begin{aligned}
& B^{*}\left(-\Delta_{N}, N, \pi_{N}\right) \sim B^{*}\left(-\Delta_{N}, N, \pi_{N}\right)-B^{*}\left(-\Delta_{N}(1+\zeta), N, \pi_{N}\right) \\
& \sim 1-B^{*}\left(\Delta_{N}, N, \pi_{N}\right) \stackrel{\sim}{*} B^{*}\left(\Delta_{N}(1+\zeta), N, \pi_{N}\right)-B^{*}\left(\Delta_{N}, N, \pi_{N}\right) \\
& \sim \Phi_{N}\left(-\Delta_{N} /\left[N \pi_{N}\left(1-\pi_{N}\right)\right]^{\frac{1}{2}}\right) \text {, }
\end{aligned}
$$

so that the logarithm of any of these behaves as

$$
\log B^{*}\left(-\Delta_{N}, N, \pi_{N}\right) \sim-\Delta_{N}{ }^{2} / 2 N \pi_{N} .
$$

Proof. The standard proof in Feller [6], pp. 168-179, for the case where $\pi_{N}$ is constant, is easily seen to apply, essentially intact, under the present assumptions. (It even yields the same conclusion with $\zeta$ replaced by $\zeta_{N}$ such that $N \pi_{N}$ / $\Delta_{N}^{2} \zeta_{N}=o(1)$, but we shall not require this stronger result.) Of course, $1-\pi_{N} \rightarrow 1$ is used in (2.2).

We shall also use (2.1) in the usual way to obtain estimates for the law of $K_{N}$, where $\pi_{N}=p$ for all $N$; this entails only multiplying the right side of (2.1) by $(1-p)^{-1}$, to obtain an estimate of $\log P\left\{N^{\frac{1}{2}} K_{N}>\Delta_{N}\right\}$.

The following lemmas use the sample df conditioning technique (as employed, e.g., in [7]), and make use only of Chebyshev's inequality and the fact that, given that $K_{M}=k>0$, the law of $M\left[T_{M}(x)+x\right]$ for $x>0$ is $B(\cdot, M(1-p)+$ $\left.M^{\frac{1}{2}} k, x /(1-p)\right)$; the corresponding unconditional law is of course $B(\cdot, M, x)$. We shall not aim at sharp hypotheses or conclusions, but merely at the forms we require. Thus, all these estimates have unconditional and various conditional versions, of which we state only the forms actually used in the sequel. Also, under the conditions of Lemma 3 , the analogue of $(2.9)$ with " $>w$ " replaced by " $<-w$ " in both events, is easily proved in the same way; as a corollary of these two results we then have, under the same conditions,

$$
\begin{array}{r}
P\left\{T_{M}((1-\delta) a)>(1-3 \delta) w \mid T_{M}(a)>w, K_{M}=k\right\} \geqq \frac{2}{3}, \\
P\left\{T_{M}((1-\delta) a)<-(1-3 \delta) w \mid T_{M}(a)<-w, K_{M}=k\right\} \geqq \frac{2}{3},
\end{array}
$$

which inequalities we shall make use of; these could have been proved directly with " $3 \delta$ " replaced by a smaller multiple of $\delta$ by repeating the argument of the last few lines of (2.6), and (with $\frac{2}{3}$ reduced to a smaller positive value) even with " $3 \delta$ " replaced by " $\delta$ " by using a different argument. We forego such repetition and unneeded elegance. The results which are required for proving the analogue of (1.14) obtained from the first half of (1.12) are proved in almost the same way, and the form of their statements will be clear; for example, from (1.12) we see that (2.4) will be replaced by considering negative deviations over $1 \leqq z \leqq 1+\delta$.

Lemma 3. Suppose $0<\delta \leqq \frac{1}{6}, a>0, M \delta w \geqq 2$, and that $M \delta w^{2} /(a+w) \geqq 32$. Then, wp 1,

$$
P\left\{\inf _{1-\delta \leqq z \leqq 1} T_{M}(z a)>(1-3 \delta) w \mid T_{M}(a)>w, K_{M}=k\right\} \geqq \frac{2}{3} .
$$


Proof. Let $Z$ be the largest value in $[1-\delta, 1]$, if there is any, for which $T_{M}(Z a-) \leqq(1-3 \delta) w$. Given the condition of (2.4) and also that $Z=z$ and $T_{M}(z a-)=(1-3 \delta) s \leqq(1-3 \delta) w$, the random function $\left\{T_{M}(x), 0 \leqq x \leqq z a\right\}$ clearly has the same law as it does under the sole condition $T_{M}(z a-)=(1-3 \delta) s$, since $Z$ is the first positive value $z$ for which $T_{M}(z a-) \leqq(1-3 \delta) w$ as one decreases $z$ from $z=1$. Thus, since $[w-(1-2 \delta) s / z] \geqq \delta w$,

$$
\begin{aligned}
P & \left\{T_{M}((1-2 \delta) a)\right. \\
& \left.\leqq(1-3 \delta) w \mid T_{M}(a)>r, K_{M}=k, Z=z, T_{M}(z a-)=(1-3 \delta) s\right\} \\
& =B(M(1-2 \delta) a+M(1-3 \delta) w, M z a+M(1-3 \delta) s,(1-2 \delta) / z) \\
& =B^{*}(M(1-3 \delta)[w-(1-2 \delta) s / z], M z a+M(1-3 \delta) s,(1-2 \delta) / z) \\
& \geqq B^{*}(M(1-3 \delta) \delta w, M z a+M(1-3 \delta) s,(1-2 \delta) / z) \geqq \frac{3}{4},
\end{aligned}
$$

this last by Chebyshev's inequality using the fact that the arguments of the last $B^{*}$ satisfy $\Delta /[N \pi(1-\pi)]^{\frac{1}{2}} \geqq\left[M \delta w^{2} / 8(a+w)\right]^{\frac{1}{2}}$. We thus have

$$
\begin{aligned}
& P\left\{\inf _{1-\delta \leqq z \leqq 1} T_{M}(z a) \leqq(1-\delta) w \mid T_{M}(a)>w, K_{M}=k\right\} \\
& P\left\{Z \text { exists, } T_{M}(Z a-) \leqq(1-3 \delta) w, T_{M}((1-2 \delta) a)\right. \\
& =\frac{\left.\leqq(1-3 \delta) w \mid T_{M}(a)>w, K_{M}=k\right\}}{P\left\{T_{M}((1-2 \delta) a) \leqq(1-3 \delta) w \mid T_{M}(a)>w, K_{M}=k,\right.} \\
& \leqq \frac{4}{3} P\left\{T_{M}((1-2 \delta) a) \leqq(1-3 \delta) w \mid T_{M}(a)>w, K_{M}=k\right\} \\
& \leqq \frac{4}{3} \sup _{\bar{s}>w} P\left\{T_{M}((1-2 \delta) a) \leqq(1-3 \delta) w \mid T_{M}(a)=\bar{s}, K_{M}=k\right\} \\
& =\frac{4}{3} \sup _{\bar{s}>w} B(M(1-2 \delta) a+M(1-3 \delta) w, M(a+\bar{s}),(1-2 \delta)) \\
& =\frac{4}{3} \sup _{\bar{s}>w} B^{*}(M(1-2 \delta)(w-\bar{s})-M \delta w, M(a+\bar{s}),(1-2 \delta)) \leqq \frac{1}{3} \text {, }
\end{aligned}
$$

the last by Chebyshev's inequality using $(1-2 \delta)(\bar{s}-w)+\delta w \geqq$ $(a+\bar{s})^{\frac{1}{2}}(a+w)^{-\frac{1}{2}} w \delta / 2$, so that from $(1.10)$ the arguments of the last $B^{*}$ satisfy $-\Delta /[N \pi(1-\pi)]^{\frac{1}{2}} \geqq\left[M \delta w^{2} / 8(a+w)\right]^{\frac{1}{2}}$. The last bound of $(2.6)$ proves Lemma 3 .

Lemma 4. If $0<\delta<1,0<a \leqq 1-p, 0<w$, then, wp 1 ,

$$
\begin{gathered}
P\left\{T_{M}(a)>w(1-\delta) \mid T_{M}{ }^{+}(a)>w\right\} \\
\geqq \frac{3}{4} \text { if }[M / a]^{\frac{1}{2}} w(\delta-a) \geqq 2 ; \\
P\left\{T_{M}(a)<-w(1-\delta) \mid T_{M}^{-}(a)>w\right\} \\
\geqq \frac{3}{4} \text { if }[M / a(1+w)]^{\frac{1}{2}} w(\delta-a) \geqq 2 ; \\
P\left\{T_{M}(a)>w(1-\delta) \mid T_{M}{ }^{+}(a)>w, K_{M}=k\right\} \\
\geqq \frac{3}{4} \quad \text { if }[M / a(1-p)]^{\frac{1}{2}} w\left(\delta-a(1-p)^{-1}\right) \geqq 2 .
\end{gathered}
$$

Proof. Let $Z^{\prime}$ be the smallest positive value for which $T_{M}{ }^{+}\left(Z^{\prime}\right)>w$, say $T_{M}{ }^{+}\left(Z^{\prime}\right)=s>w$. Then, for $z<a$,

$$
\begin{aligned}
& P\left\{T_{M}(a)>w(1-\delta) \mid Z^{\prime}=z, T_{M}(z)=s\right\} \\
& \quad=1-B(M[a+w(1-\delta)-z-s], M(1-z-s),(a-z) /(1-z))
\end{aligned}
$$




$$
\begin{aligned}
= & 1-B^{*}(-M w \delta+M[-(s-w)(1-a)+(a-z) w] /(1-z), \\
& M(1-z-s),(a-z) /(1-z)) \\
\geqq & 1-B^{*}(-M w(\delta-a), M(1-z-s),(a-z) /(1-z)) \geqq \frac{3}{4}
\end{aligned}
$$

by Chebyshev's inequality, since the arguments of the last $B^{*}$ 管 satisfy $-\Delta /[N \pi(1-\pi)]^{\frac{1}{2}} \geqq M^{\frac{1}{2}} a^{-\frac{1}{2}} w(\delta-a)$. This yields the first line of $(2.7)$. The other lines are proved analogously.

Lemma 5. Under the conditions of Lemma 3, wp 1,

$$
\begin{aligned}
P\left\{\inf _{1-\delta \leqq z \leqq 1} T_{M}(z a)>(1-3 \delta) w \mid K_{M}\right. & =k\} \\
& \geqq \frac{2}{3} P\left\{T_{M}(a)>w \mid K_{M}=k\right\} .
\end{aligned}
$$

Under the conditions of Lemma 4,

$$
\begin{aligned}
P\left\{T_{M}^{+}(a)>w\right\} & \leqq \frac{4}{3} P\left\{T_{M}(a)>w(1-\delta)\right\} \\
P\left\{T_{M}^{-}(a)>w\right\} & \leqq \frac{4}{3} P\left\{T_{M}(a)<-w(1-\delta)\right\} \\
P\left\{T_{M}^{+}(a)>w \mid K_{n}=k\right\} & \leqq \frac{4}{3} P\left\{T_{M}(a)>w(1-\delta) \mid K_{n}=k\right\} \text { wp } 1 .
\end{aligned}
$$

Proof. (2.9) follows from (2.4) and $P\{A \mid C\} \geqq P\{A \mid B C\} P\{B \mid C\}$. (2.10) follows from (2.7) and $P\{A \mid C\} \leqq P\{B \mid C\} / P\{B \mid A C\}$ (with $C=$ sample space in first two lines), just as used in the first three lines of (2.6).

3. Lower class result. In this section we shall prove the first half of (1.14). Throughout this section $\lambda$ is fixed, $0<\lambda<1$, and we write $\epsilon=1-\lambda$. We define $S_{n_{r}, n}$ for $n_{r}<n \leqq n_{r+1}$, as the sample df based on $\left(X_{n_{r}+1}, X_{n_{r}+2}, \cdots, X_{n}\right)$; similarly, $K_{n_{r}, n}=\left(n-n_{r}\right)^{\frac{1}{2}}\left(p-S_{n_{r}, n}(p)\right)$, and $T_{n_{r}, n}$ (with or without superscript) is then obtained by replacing $S_{n}$ and $K_{n}$ by $S_{n_{r}, n}$ and $K_{n_{r}, n}$ in (1.11). $\gamma$ is any value which satisfies

$$
\begin{aligned}
& \gamma^{\frac{1}{3}}>9, \\
& -\gamma^{-\frac{1}{3}}+(1-4 \epsilon / 5)(\gamma-1) / \gamma>1-\epsilon, \\
& -(7 / \gamma)^{\frac{1}{2}}+(1-\epsilon / 25)(\gamma-1) / \gamma>1-\epsilon / 20 ;
\end{aligned}
$$

it is clear that such a $\gamma$ exists, and the somewhat redundant form of (3.1) is pointed toward its use in the sequel. We shall repeatedly use the fact that $\lim _{r \rightarrow \infty} n_{r+1} / n_{r}=\gamma$.

We define the following events:

$$
\begin{aligned}
L_{r+1}^{\prime} & =\left\{1-\epsilon / 20<K_{n_{r+1}} / n_{r+1}^{\frac{1}{2}} h_{n_{r+1}}<1\right\}, \\
L_{r+1}^{\prime \prime} & =\left\{\inf _{1-\epsilon / 10 \leqq x \leqq 1} T_{n_{r+1}}\left(x h_{n_{r+1}}\right)>q_{n_{r+1}}(1-\epsilon)\right\} \\
G_{r}^{\prime} & =\left\{\left|K_{n_{r}}\right|<2 \sigma_{p}\left(\log \log n_{r}\right)^{\frac{1}{2}}\right\} \\
G_{r}^{\prime \prime} & =\left\{T_{n_{r}}^{-}\left(h_{n_{r+1}}\right) \leqq \gamma^{\frac{2}{3}} q_{n_{r+1}}\right\} \\
G_{r, r+1}^{\prime} & =\left\{1-\epsilon / 30<K_{n_{r}, n_{r+1}} /\left(\mathrm{n}_{r+1}-n_{r}\right)^{\frac{1}{2}} h_{n_{r+1}}<1-\epsilon / 60\right\} \\
G_{r, r+1}^{\prime \prime} & =\left\{\inf _{1-\epsilon / 10 \leqq x \leqq 1} T_{n_{r}, n_{r+1}}\left(x h_{n_{r+1}}\right)>(1-3 \epsilon / 10)(1-\epsilon / 2) q_{n_{r+1}}\right\} .
\end{aligned}
$$


We shall prove that the following inclusions hold for sufficiently large $r$ (depending only on $p, \lambda, \gamma)$ :

$$
\begin{gathered}
L_{r+1}^{\prime} \cap L_{r+1}^{\prime \prime} \subset A_{n_{r+1}}(\lambda), \\
G_{r}^{\prime} \cap G_{r, r+1}^{\prime} \subset L_{r+1}^{\prime}, \\
G_{r}^{\prime \prime} \cap G_{r, r+1}^{\prime \prime} \subset L_{r+1}^{\prime \prime},
\end{gathered}
$$

and we shall also prove that

$$
\begin{aligned}
& G_{r}^{\prime} \text { occurs for a.a.r, wp } 1, \\
& G_{r}^{\prime \prime} \text { occurs for a.a.r, wp 1, } \\
& G_{r, r+1}^{\prime} \cap G_{r, r+1}^{\prime \prime} \text { occurs i.o., wp } 1 .
\end{aligned}
$$

Clearly, (1.14) follows from (3.3)-(3.8).

First, note that

$$
L_{r+1}^{\prime} \cap L_{r+1}^{\prime \prime} \quad \text { entails } \quad T_{n_{r+1}}\left(n_{r+1}^{-\frac{1}{2}} K_{n_{r+1}}-z\right)>\lambda q_{n_{r+1}}
$$

for $0<z<h_{n_{r+1}} \epsilon / 20$; in particular, since $q_{n}=o\left(h_{n}\right)$, this holds for $z=\lambda q_{n_{r+1}}$ if $r$ is sufficiently large. Reference to (1.12) yields (3.3).

To prove (3.4), we note that

$$
n_{r+1}^{\frac{1}{2}} K_{n_{r+1}}=n_{r}^{\frac{1}{2}} K_{n_{r}}+\left(n_{r+1}-n_{r}\right)^{\frac{1}{2}} K_{n_{r}, n_{r+1}} .
$$

Under $G_{r}^{\prime}$,

$$
n_{r+1}^{-1} h_{n_{r+1}}^{-1} n_{r}^{\frac{1}{2}}\left|K_{n_{r}}\right| \sim\left|K_{n_{r}}\right| / \sigma_{p}\left[(2 \gamma / 3) \log \log n_{r}\right]^{\frac{1}{2}}<(6 / \gamma)^{\frac{1}{2}}
$$

so that the left side of $(3.10)$ is $<(7 / \gamma)^{\frac{1}{2}}$ for all large $r$. Under $G_{r, r+1}^{\prime}$,

$$
\begin{aligned}
n_{r+1}^{-1} h_{n_{r+1}}^{-1}\left(n_{r+1}-n_{r}\right)^{\frac{1}{2}} K_{n_{r}, n_{r+1}} & \sim[(\gamma-1) / \gamma] K_{n_{r}, n_{r+1}} /\left(n_{r+1}-n_{r}\right)^{\frac{1}{2}} h_{n_{r+1}} \\
& =[(\gamma-1) / \gamma][1-(1+\theta) \epsilon / 60]
\end{aligned}
$$

where $0<\theta<1$, so that for all large $r$ the left side is $[(\gamma-1) / \gamma] \times$ $[1-(1+\theta) \epsilon / 50]$. Dividing both sides of (3.9) by $n_{r+1} h_{n_{r+1}}$ and invoking these consequences of (3.10)-(3.11) together with the third line of (3.1) (which last also implies that $\left.(7 / \gamma)^{\frac{1}{2}}+(1-\epsilon / 50)(\gamma-1) / \gamma<1\right)$, we obtain (3.4).

Next, we apply

$$
n_{r} T_{n_{r}}+\left(n_{r+1}-n_{r}\right) T_{n_{r}, n_{r+1}}=n_{r+1} T_{n_{r+1}}
$$

to (3.2) and see that (3.5) would follow from

$$
-n_{r} \gamma^{\frac{2}{3}}+\left(n_{r+1}-n_{r}\right)(1-3 \epsilon / 10)(1-\epsilon / 2)>n_{r+1}(1-\epsilon) .
$$

Dividing all members of (3.13) by $n_{r+1}$ and applying the second line of (3.1), we obtain (3.5) for all large $r$.

As for (3.6), it is of course a consequence of the ordinary law of the iterated logarithm for Bernoulli random variables.

To prove (3.7), we use the second line of (2.10) with $M=n_{r}, a=h_{n_{r+1}}$, $w=\gamma^{\frac{2}{3}} q_{n_{r+1}}$, and $\delta=\frac{1}{2}$, so that we have $[M / a(1+w)]^{\frac{1}{2}} w(\delta-a) \rightarrow \infty$ as $r \rightarrow \infty$ 
and conclude that, for all large $r$,

$$
\begin{aligned}
P\left\{\overline{G_{r}{ }^{\prime \prime}}\right\} & =P\left\{T_{n_{r}}^{-}\left(h_{n_{r+1}}\right)>\gamma^{\frac{3}{3}} q_{n_{r+1}}\right\} \\
& \leqq \frac{4}{3} P\left\{T_{n_{r}}\left(h_{n_{r+1}}\right)<-2^{-1} \gamma^{\frac{3}{3}} q_{n_{r+1}}\right\} .
\end{aligned}
$$

Applying (2.2) to $B^{*}(-\Delta, N, \pi)$ with $N=n_{r}, \pi=h_{n_{r+1}}$, and $\Delta=2^{-1} \gamma^{\frac{3}{3}} n_{r} q_{n_{r+1}}$, so that $\Delta^{3} / N^{2} \pi^{2} \rightarrow 0$ and $\Delta^{2} / N \pi \sim \gamma^{\frac{1}{3}} 3^{-1} \log r$ (making Lemma 2 applicable) as $r \rightarrow \infty$, we obtain, for all sufficiently large $r$, from the first line of (3.1),

$$
\log P\left\{\overline{G_{r}^{\prime \prime}}\right\}<-\frac{4}{3} \log r .
$$

Hence $\sum_{r} P\left\{\overline{G_{r}{ }^{\prime \prime}}\right\}<\infty$, which by the Borel-Cantelli Lemma yields (3.7).

It remains to prove (3.8). Applying the classical form of (2.1) for $1-B^{*}$ in the case $\pi_{n} \equiv p, N=n_{r+1}-n_{r}, \Delta_{N}=(1-\epsilon / 30)\left(n_{r+1}-n_{r}\right) h_{n_{r+1}}$, $1+\zeta=(1-\epsilon / 60) /(1-\epsilon / 30)$, as described in the second paragraph below (2.2), we have

$$
\log P\left\{G_{r, r+1}^{\prime}\right\} \sim-(3 \gamma)^{-1}(\gamma-1)(1-\epsilon / 30)^{2} \log r
$$

as $r \rightarrow \infty$. From (2.9) with $M=n_{r+1}-n_{r}, \delta=\epsilon / 10, a=h_{n_{r+1}}$, $w=(1-\epsilon / 2) q_{n_{r+1}}$ (so that the conditions of Lemma 3 hold for large $r$ ), we have

$$
\begin{aligned}
P\left\{G_{r, r+1}^{\prime \prime} \mid K_{n_{r}, n_{r+1}}\right. & =k\} \\
& \geqq \frac{2}{3} P\left\{T_{n_{r}, n_{r+1}}\left(h_{n_{r+1}}\right)>(1-\epsilon / 2) q_{n_{r+1}} \mid K_{n_{r}, n_{r+1}}=k\right\}
\end{aligned}
$$

for $r$ sufficiently large. Applying $(2.2)$ to $1-B^{*}$ for $N=(1-p)\left(n_{r+1}-n_{r}\right)$ $+\left(n_{r+1}-n_{r}\right)^{\frac{1}{2}} k, \pi_{N}=(1-p)^{-1} h_{n_{r+1}}, \Delta_{N}=\left(n_{r+1}-n_{r}\right)(1-\epsilon / 2) q_{n_{r+1}}$ (so that the conditions of Lemma 2 are satisfied), we have, as $r \rightarrow \infty$, uniformly in $k$ satisfying $1-\epsilon / 30<k /\left(n_{r+1}-n_{r}\right)^{\frac{1}{2}} h_{n_{r+1}}<1-\epsilon / 60$,

$$
\begin{aligned}
\log P\left\{T_{n_{r}, n_{r+1}}\left(h_{n_{r+1}}\right)>(1-\epsilon / 2)\right. & \left.q_{n_{r+1}} \mid K_{n_{r}, n_{r+1}}=k\right\} \\
& \sim-2(\gamma-1)(3 \gamma)^{-1}(1-\epsilon / 2)^{2} \log r .
\end{aligned}
$$

From (3.16), (3.17), and (3.18), we have for $r$ sufficiently large,

$$
\log P\left\{G_{r, r+1}^{\prime} \cap G_{r, r+1}^{\prime \prime}\right\} \geqq-(1-\epsilon / 30)^{2} \log r,
$$

so that $\sum_{r} P\left\{G_{r, r+1}^{\prime} \cap G_{r, r+1}^{\prime \prime}\right\}=\infty$. The events $\left\{G_{r, r+1}^{\prime} \cap G_{r, r+1}^{\prime \prime}, r=1,2, \cdots\right\}$ being independent, the Borel-Cantelli Lemma yields (3.8), completing the proof of the first half of (1.14).

4. Upper class result. In this section we prove the second half of (1.14). We use the notation of the first paragraph of Section 3, except that now $\lambda=1+\epsilon>1$ and (3.1) is replaced by fixing $\eta=\gamma-1$ at any positive value satisfying

$$
\begin{aligned}
& \eta<2^{-6} 3^{-2} \epsilon^{2} \\
& 1+8 \eta^{\frac{1}{2}}<(.98) 2^{\frac{5}{2}} 3^{-\frac{3}{2}} \\
& \left(1+10 \eta^{\frac{1}{2}}\right)\left(1-\eta^{\frac{1}{2}}\right)^{-1}<(1+\epsilon / 2) \\
& \eta<10^{-4}
\end{aligned}
$$


We now define

$$
\begin{aligned}
S= & \left\{L: 2^{-1} \leqq L \leqq 2+4 \eta^{\frac{1}{2}} ; L=\text { integer } \times \eta^{\frac{1}{2}}\right. \text { or } \\
& \left.L=2^{-1} \text { or } L=2+4 \eta^{\frac{1}{2}}\right\}, \\
I_{r}= & \left\{n: n_{r}<n \leqq n_{r+1}\right\}, \\
J_{r}= & \left\{\left|K_{n}\right|<2 \sigma_{p}\left(\log \log n_{r}\right)^{\frac{1}{2}}, n \varepsilon I_{r}\right\}, \\
H_{r}= & \left\{\left|K_{n}-K_{n_{r}}\right|<4 \eta^{\frac{1}{2}} \sigma_{p}\left(\log \log n_{r}\right)^{\frac{1}{2}}, n \varepsilon I_{r}\right\}, \\
D_{r}= & \left\{\left(n-n_{r}\right) T_{n_{r}, n}+\left(3 h_{n_{r}}\right)<2^{-1} \epsilon n_{r} q_{n_{r}}, n \varepsilon I_{r}\right\}, \\
E_{r}= & \left\{\left|K_{n_{r}}\right|<2^{-1} \sigma_{p}\left(\log \log n_{r}\right)^{\frac{1}{2}} ; A_{n}(\lambda) \quad \text { occurs for some } n \varepsilon I_{r}\right\}, \\
B_{L, r}= & \left\{\left|1-K_{n_{r}} / L \sigma_{p}\left(\log \log n_{r}\right)^{\frac{1}{2}}\right|<\eta^{\frac{1}{2}}\right\} \quad \text { where } 2^{-1} \leqq L \leqq 2+4 \eta^{\frac{1}{2}}, \\
C_{L, r}= & \left\{T_{n_{r}}^{+}\left(\left(1+10 \eta^{\frac{1}{2}}\right) 2^{-\frac{1}{2}} 3^{\frac{1}{2}} L h_{n_{r}}\right)>(1+\epsilon / 2) q_{n_{r}}\right\}, \\
C_{L, n}^{\prime}= & \left\{T_{n}^{+}\left(\left(1+10 \eta^{\frac{1}{2}}\right) 2^{-\frac{1}{2}} 3^{\frac{1}{2}} L h_{n_{r}}\right)>\lambda q_{n}\right\} \quad \text { where } n \varepsilon I_{r} .
\end{aligned}
$$

We shall prove that

(4.3) $J_{r}$ occurs for a.a.r, wp 1,

(4.4) $H_{r}$ occurs for a.a.r, wp 1 ,

(4.5) $D_{r}$ occurs for a.a.r, wp 1 ,

(4.6) $\bar{E}_{r}$ occurs for a.a.r, wp 1 ,

(4.7) $B_{L, R} \cap C_{L, R}$ occurs only finitely often wp 1, for each fixed $L \varepsilon S$,

and we shall also prove the following hold for all sufficiently large $r$ (depending only on $p, \lambda, \eta)$ :

$$
\begin{gathered}
B_{L, r} \cap H_{r} \cap A_{n}(\lambda) \subset C_{L, n}^{\prime} \text { for each } n \varepsilon I_{r}, \quad L \varepsilon S, \\
\mathrm{D}_{r} \cap\left(\mathrm{U}_{n \varepsilon I_{r}} C_{L, n}^{\prime}\right) \subset C_{L, r} \text { for each } L \varepsilon S, \\
J_{r} \cap H_{r} \cap \tilde{E}_{r} \cap A_{n}(\lambda) \subset \mathrm{U}_{L \varepsilon S} B_{L, r} \text { for } n \varepsilon I_{r} .
\end{gathered}
$$

From (4.3), (4.4), (4.6), and (4.10), and from the finiteness of $S$, it then follows that, except on a set of probability zero, $A_{n}(\lambda)$ occurs i.o. only if, for some $L$ in $S$, the event $B_{L, r} \cap H_{r} \cap\left(\mathrm{U}_{n \varepsilon I_{r}} A_{n}(\lambda)\right)$ occurs i.o. This occurrence and (4.5) imply, by (4.8)-(4.9), that $B_{L, r} \cap C_{L, r}$ occurs i.o. for some $L \varepsilon S$. Thus, (4.7) yields the desired second half of (1.14).

(4.3) is a consequence of the ordinary law of the iterated logarithm for Bernoulli variables and the fact that $\log \log n \sim \log \log n_{r}$ for $n \varepsilon I_{r}$.

To prove (4.4), let $Z_{i}^{\prime}$ be the indicator of the event $\left\{X_{i} \leqq p\right\}$, and let $Z_{i}=Z_{i}^{\prime}-p$. It is well known (e.g., [6], p. 192, equation (5.7)) that there is a finite constant $b_{p}$ such that, for all $\alpha_{r}>0$,

$$
P\left\{\max _{n \varepsilon I_{r}}\left|\sum_{n_{r}+1}^{n} Z_{j}\right|>\alpha_{r}\right\} \leqq b_{p} P\left\{\left|\sum_{j_{\varepsilon} I_{r}} Z_{j}\right|>\alpha_{r}\right\} .
$$


Putting $\alpha_{r}=2 \eta^{\frac{1}{2}} \sigma_{p}\left(n_{r} \log \log n_{r}\right)^{\frac{1}{2}}$ and using the classical form of $(2.1)$. as described in the second paragraph below (2.2) with $N=n_{r+1}-n_{r}, \pi_{N}=p$, $\Delta_{N}=\alpha_{r}$, and the fact that $n^{-\frac{1}{2}}<n_{r}^{-\frac{1}{2}}$ for $n \varepsilon I_{r}$, we have

(4.12) $\log P\left\{\max _{n \varepsilon I_{r}} n^{-\frac{1}{2}}\left|\sum_{n_{r}+1}^{n} Z_{j}\right|>2 \eta^{\frac{1}{2}} \sigma_{p}\left(\log \log n_{r}\right)^{\frac{1}{2}}\right\}$

$$
\leqq-2(1+o(1)) \log r .
$$

Hence, by the Borel-Cantelli Lemma,

$$
\max _{n \varepsilon I_{r}} n^{-\frac{1}{2}}\left|\sum_{n_{r}+1}^{n} Z_{j}\right| \leqq 2 \eta^{\frac{1}{2}} \sigma_{p}\left(\log \log n_{r}\right)^{\frac{1}{2}} \text { for a.a.r, wp } 1 \text {. }
$$

Clearly,

$$
\begin{aligned}
K_{n_{r}}-K_{n} & =n^{-\frac{1}{2}} \sum_{1}^{n} Z_{i}-n_{r}^{-\frac{1}{2}} \sum_{1}^{n_{r}} Z_{i} \\
& =n^{-\frac{1}{2}} \sum_{n_{r}+1}^{n} Z_{i}+K_{n_{r}}\left[\left(n_{r} / n^{2}\right)^{\frac{1}{2}}-1\right] .
\end{aligned}
$$

Since $0<1-\left(n_{r} / n\right)^{\frac{1}{2}}<\eta$ for $n \varepsilon I_{r}$ and all large $r$, we conclude from (4.13), (4.14), the validity of (4.3) with $I_{r}$ replaced by $\left\{n_{r}\right\}$, and the fourth line of (4.1) (which implies $\eta<\eta^{\frac{1}{2}}$ ) that (4.4) holds.

We turn to the proof of (4.5). If $\bar{D}_{r}$ occurs, let $\nu$ be the first integer $n \varepsilon I_{r}$ for which the inequality defining $D_{r}$ fails to hold, and let $Z$ be the smallest positive value for which

$$
\left(\nu-n_{r}\right) T_{n_{r}, \nu}\left(3 Z h_{n_{r}}\right) \geqq 2^{-1} \epsilon n_{r} q_{n_{r}} .
$$

Now, for all large $r$, uniformly in $n \varepsilon I_{r}$ and $z \varepsilon(0,1]$,

$$
\begin{aligned}
P\left\{\left(n_{r+1}-n\right) T_{n, n_{r+1}}\left(3 z h_{n_{r}}\right)\right. & \left.<-4^{-1} \epsilon n_{r} q_{n_{r}} \mid \bar{D}_{r}, \nu=n, Z=z\right\} \\
= & B^{*}\left(-4^{-1} \epsilon n_{r} q_{n_{r}}-,\left(n_{r+1}-n\right), 3 z h_{n_{r}}\right) \leqq \frac{1}{4},
\end{aligned}
$$

by Chebyshev's inequality and the fact that the arguments of $B^{*}$ in (4.16) satisfy $\Delta^{2} / N \pi \rightarrow \infty$ with $r$, uniformly. (If $n=n_{r+1}$, the probability in (4.16) is zero.) Consideration of (4.15) together with the event complementary to that of (4.16) yields, for large $r$,

$$
P\left\{\left(n_{r+1}-n_{r}\right) T_{n_{r}, n_{r+1}}^{+}\left(3 h_{n_{r}}\right)>4^{-1} \epsilon n_{r} q_{n_{r}} \mid \bar{D}_{r}\right\} \geqq \frac{3}{4}
$$

and hence, first using the familiar argument used to prove (2.10), and then using the first line of (2.10) itself with $\delta=\frac{1}{2}$,

$$
\begin{aligned}
\log P\left\{\bar{D}_{r}\right\} & \leqq \log \left[\frac{4}{3} P\left\{\left(n_{r+1}-n_{r}\right) T_{n_{r}, n_{r+1}}^{+}\left(3 h_{n_{r}}\right)>4^{-1} \epsilon n_{r} q_{n_{r}}\right\}\right] \\
& \leqq \log \left[(16 / 9)\left\{1-B^{*}\left(8^{-1} \epsilon n_{r} q_{n_{r}}, n_{r+1}-n_{r}, 3 h_{n_{r}}\right)\right\}\right] \\
& \sim-\epsilon^{2} \eta^{-1} 2^{-5} 3^{-2} \log r
\end{aligned}
$$

the last coming from an application of (2.2). Thus, (4.5) follows from the first line of (4.1) and the Borel-Cantelli Lemma for $\left\{\bar{D}_{r}\right\}$.

To prove (4.6), we first note that for large $r$ the events

$$
Q_{r}=\left\{T_{n_{r}}^{+}\left(2^{-1}\left(1+8 \eta^{\frac{1}{2}}\right) \sigma_{p} n_{r}^{-\frac{1}{2}}\left(\log \log n_{r}\right)^{\frac{1}{2}}\right) \leqq q_{n_{r}}\right\}
$$


satisfy, by (2.10) with $\delta=.01$ and by (2.2),

$$
\log P\left\{\bar{Q}_{r}\right\}<-(.98) 2^{\frac{5}{2}} 3^{-\frac{3}{2}}\left(1+8 \eta^{\frac{1}{2}}\right)^{-1} \log r,
$$

so that the second line of (4.1) and the Borel-Cantelli Lemma for $\left\{\widetilde{Q}_{r}\right\}$ yield

$$
Q_{r} \quad \text { occurs for a.a.r, wp } 1 .
$$

Note that $E_{r} \cap H_{r}$ entails $\left|K_{n}\right|<2^{-1}\left(1+8 \eta^{\frac{1}{2}}\right) \sigma_{p}\left(\log \log n_{r}\right)^{\frac{1}{2}}$ for $n \varepsilon I_{r}$, so that by (1.12) and $n^{-\frac{1}{2}}<n_{r}^{-\frac{1}{2}}$ we see that $E_{r} \cap H_{r} \cap A_{n}(\lambda)$ entails either

$$
T_{n}^{+}\left(2^{-1}\left(1+8 \eta^{\frac{1}{2}}\right) \sigma_{p} n_{r}^{-\frac{1}{2}}\left(\log \log n_{r}\right)^{\frac{1}{2}}\right)>\lambda q_{n}
$$

or else (if $0<K_{n} \leqq \lambda n^{-\frac{1}{2}} q_{n}$ )

$$
T_{n}{ }^{+}\left(-\lambda q_{n}\right)>\lambda q_{n}
$$

However, $Q_{r} \cap D_{r}$ entails, for $n \varepsilon I_{r}$,

$$
T_{n}{ }^{+}\left(2^{-1}\left(1+8 \eta^{\frac{1}{2}}\right) \sigma_{p} n_{r}^{-\frac{1}{2}}\left(\log \log n_{r}\right)^{\frac{1}{2}}\right)<(1+\epsilon / 2)\left(n_{r} / n\right) q_{n_{r}}<\lambda q_{n},
$$

so that (4.5) and (4.21) imply that, wp 1, the event (4.22) occurs for only finitely many $n$. The event (4.23), although it involves a negative argument, is even easier to handle since $q_{n}$ is small compared with the argument of $T_{n}{ }^{+}$in (4.22); for example, one can consider the random variables $1-X_{i}$, as described below (1.14), and use the obvious analogues of (4.5) and (4.19); we conclude that, wp 1, (4.23) also occurs for only finitely many $n$. This last is thus true of $E_{r} \cap H_{r} \cap A_{n}(\lambda)$, and by (4.4) this yields (4.6).

As for (4.7), by the classical form of (2.1) for $1-B^{*}$ (described in the second paragraph below $(2.2))$ with $\pi_{N} \equiv p, N=n_{r}, \Delta_{N}=L \sigma_{p}\left(n_{r} \log \log n_{r}\right)^{\frac{1}{2}}\left[1-\eta^{\frac{1}{2}}\right]$, $\zeta+1=\left[1+\eta^{\frac{1}{2}}\right] /\left[1-\eta^{\frac{1}{2}}\right]$, we have

$$
\log P\left\{B_{L, r}\right\} \sim-2^{-1} L^{2}\left(1-\eta^{\frac{1}{2}}\right)^{2} \log r .
$$

On the other hand, uniformly for $\left|1-k / L \sigma_{p}\left(\log \log n_{r}\right)^{\frac{1}{2}}\right|<\eta^{\frac{1}{2}}$, we have, from (2.2) and the third line of $(2.10)$ with $1-\delta=(1+\epsilon / 4) /(1+\epsilon / 2)$, for $r$ large,

$$
\begin{aligned}
\log P\left\{C_{L, r} \mid K n_{r}=\right. & k\} \\
& \leqq \\
& \log \left[\frac { 4 } { 3 } \left\{1-B^{*}\left((1+\epsilon / 4) n_{r} q_{n_{r}}, n_{r}(1-p)+n_{r}^{\frac{1}{2}} k\right.\right.\right. \\
& \left.\left.\left.(1-p)^{-1}\left(1+10 \eta^{\frac{1}{2}}\right)\left(\frac{3}{2}\right)^{\frac{1}{2}} L h_{n_{r}}\right)\right\}\right] \\
\sim & -L^{-1}\left(1+10 \eta^{\frac{1}{2}}\right)^{-1}(1+\epsilon / 4)^{2}\left(\frac{2}{3}\right)^{\frac{3}{2}} \log r .
\end{aligned}
$$

Hence, writing $\bar{L}=L\left(1-\eta^{\frac{1}{2}}\right)$ and $\psi^{\frac{3}{2}}=(1+\epsilon / 2)\left(1-\eta^{\frac{1}{2}}\right)\left(1+10 \eta^{\frac{1}{2}}\right)^{-1}$, so that $\psi>1$ by the third line of (4.1), we have, for large $r$,

$$
\begin{aligned}
-\log P\left\{B_{L, R} \cap C_{L, R}\right\} / \log r> & 2^{-1} \bar{L}^{2}+(2 \psi / 3)^{\frac{3}{2}} \bar{L}^{-1} \\
& \geqq \min _{\alpha>0}\left[2^{-1} \alpha^{2}+(2 \psi / 3)^{\frac{3}{2}} \alpha^{-1}\right]=\psi>1 .
\end{aligned}
$$

The Borel-Cantelli Lemma now yields (4.7).

Turning to (4.8), we see that $B_{L, r} \cap H_{r}$ entails, for $n \varepsilon I_{r}$, 


$$
n^{-\frac{1}{2}} K_{n}<n^{-\frac{1}{2}} \sigma_{p}\left(\log \log n_{r}\right)^{\frac{1}{2}}\left[\left(1+\eta^{\frac{1}{2}}\right) L+4 \eta^{\frac{1}{2}}\right]<\left(1+10 \eta^{\frac{1}{2}}\right) 2^{-\frac{1}{2}} 3^{\frac{1}{2}} L h_{n_{r}},
$$

since $n>n_{r}$ and $L \geqq \frac{1}{2}$; and similarly, it entails, for large $r$,

$$
n^{\frac{1}{2}} K_{n}-\lambda q_{n}>\gamma^{-1}\left(1-10 \eta^{\frac{1}{2}}\right) 2^{-\frac{1}{2}} 3^{\frac{1}{2}} L h_{n_{r}}-\lambda q_{n}>0
$$

by the fourth line of $(4.2)$ and the fact that $q_{n}=o\left(h_{n_{r}}\right)$. Hence, (1.12), (4.28), and (4.29) yield (4.8).

As for $(4.9)$, since $3>\left(1+10 \eta^{\frac{1}{2}}\right) 2^{-\frac{1}{2}} 3^{\frac{1}{2}} L$ for $L \leqq 2+4 \eta^{\frac{1}{2}}$ (by the fourth line of (4.1)), the occurrence of $D_{r} \cap C_{L, n}^{\prime}$ for some $n \varepsilon I_{r}$ entails

$$
\begin{aligned}
n_{r} T_{n_{r}}^{+}\left(\left(1+10 \eta^{\frac{1}{2}}\right) 2^{-\frac{1}{2}} 3^{\frac{1}{2}} L h_{n_{r}}\right) & >n \lambda q_{n}-2^{-1} \epsilon n_{r} q_{n_{r}} \\
& >(1+\epsilon / 2) n_{r} q_{n_{r}}
\end{aligned}
$$

for large $r$, since $n q_{n}$ is increasing for $n \geqq 3$. This is (4.9).

Finally, if $n \varepsilon I_{r}$, then $A_{n}(\lambda) \cap \bar{E}_{n_{r}} \cap H_{r}$ implies $K_{n_{r}} \geqq 2^{-1} \sigma_{p}\left(\log \log n_{r}\right)^{\frac{1}{2}}$, since $4 \eta^{\frac{1}{2}}<2^{-1}$ by the last line of (4.1); on the other hand, $J_{r} \cap H_{r}$ implies $K_{n_{r}}<\left(2+4 \eta^{\frac{1}{2}}\right) \sigma_{p}\left(\log \log n_{r}\right)^{\frac{1}{2}}$. This proves (4.10)and completes the proof of the second half of (1.14).

5. Other proofs. We turn first to the proof of Theorem 3. The lower class result is of course implied by Theorem 1. We shall not prove the upper class result in detail, since the proof is very much like that of Section 4 for Theorem 1; instead, we shall merely indicate why only minor modifications in the latter proof are needed. As before, we assume the $X_{i}$ are uniformly distributed. Denote by $K_{n}(p)$ and $T_{n}(x, p)$ the random variables defined in (1.5) and (1.11). Then, clearly,

$$
\begin{aligned}
K_{n}\left(p+n^{-\frac{1}{2}} t\right) & =K_{n}(p)-n^{\frac{1}{2}} T_{n}\left(n^{-\frac{1}{2}} t, p\right), \\
T_{n}\left(x, p+n^{-\frac{1}{2}} t\right) & =T_{n}\left(x+n^{-\frac{1}{2}} t, p\right)-T_{n}\left(n^{-\frac{1}{2}} t, p\right)
\end{aligned}
$$

Hence, the second half of (1.12) implies that $R_{n}\left(p+n^{-\frac{1}{2}} t\right)<-\lambda q_{n}$ if and only if (except for a set of zero probability)

$$
\begin{aligned}
\lambda q_{n} \leqq & T_{n}\left(n^{-\frac{1}{2}} K_{n}\left(p+n^{-\frac{1}{2}} t\right)-\lambda q_{n}, p+n^{-\frac{1}{2}} t\right) \\
& =T_{n}\left(n^{-\frac{1}{2}} t+n^{-\frac{1}{2}} K_{n}(p)-T_{n}\left(n^{-\frac{1}{2}} t, p\right)-\lambda q_{n}, p\right)-T_{n}\left(n^{-\frac{1}{2}} t, p\right) .
\end{aligned}
$$

Now, the techniques used earlier can easily be employed to prove that, for $c>(2 \tau)^{\frac{1}{2}}$

$$
P\left\{T_{n}^{*}\left(n^{-\frac{1}{2}} \tau\right)>c n^{-3 / 4}(\log \log n)^{\frac{1}{2}} \text { i.o. }\right\}=0 .
$$

Since $n^{-3 / 4}(\log \log n)^{\frac{1}{2}}=o\left(q_{n}\right)$, the proof that, for $\lambda>1$, wp 1 there are only finitely many $n$ for which (5.2) occurs for some $t$ (depending on $n$ and the sample sequence) in $[0, \tau]$ essentially reduces to the upper class proof for Theorem 1, if one takes note of the fact that it is a uniform behavior of $T_{n}$ over an interval (e.g., in $(4.7)$ ) which is actually proved in Section 4 , and not just (1.12). In fact, it is not very difficult to see that one can even replace $\tau$ in the above by any non- 
decreasing sequence $\left\{\tau_{n}\right\}$ satisfying

$$
\tau_{n}=o\left((\log \log n)^{\frac{1}{2}}\right),
$$

since (5.3) is then still satisfied with $c$ replaced by $2 \tau_{n}^{\frac{1}{2}}$, and one thus obtains $T_{n}{ }^{*}\left(n^{-\frac{1}{2}} \tau_{n}\right)=o\left(q_{n}\right)$ wp 1 , as well as $t=o\left(K_{n}\right)$ for the crucial range of $K_{n}$ in (5.2); thus, $T_{n}\left(n^{-\frac{1}{2}} t, p\right)$ can still be added to both the first and last expression of (5.2) without appreciably changing the expression $\lambda q_{n}$ on the left. Negative values of $K_{n}\left(p+n^{-\frac{1}{2}} t\right)$ or of $t$ are treated similarly.

We turn now to the proof of Theorem 2. As before, by Lemma 1 we can and do assume the $X_{i}$ uniform. For fixed value $k_{n}$ of $K_{n} \equiv K_{n}(p)$, we shall for brevity treat in detail only the case $m=2, t_{1}=0, t_{2}>0$, and write $\left|k_{n}\right| t_{2}=s$ (in the notation just above (1.7)) ; it will be clear how larger values of $m$ and arbitrary values of the $t_{i}$ can be treated by a repetition of our steps. We shall also consider only the case $k_{n}>0$, since the complementary case is handled in the same way.

Let $u_{0}$ and $u_{1}$ be fixed real numbers. By (1.12) and (5.2) with $-\lambda q_{n}$ replaced by $n^{-3 / 4} u_{1}$, we see that, given that $K_{n}(p)=k_{n}$, the event $U_{n}(s)<u_{1}$ is equiva$1^{\text {ent to }}$

$$
T_{n}\left(n^{-\frac{1}{2}}\left(s+k_{n}\right)-T_{n}\left(n^{-\frac{1}{2}} s, p\right)+n^{-3 / 4} u_{1}, p\right)-T_{n}\left(n^{-\frac{1}{2}} s, p\right)>-n^{-3 / 4} u_{1} .
$$

The event $U_{n}(0)<u_{0}$ is of course

$$
T_{n}\left(n^{-\frac{1}{2}} k_{n}+n^{-3 / 4} u_{0}, p\right)>-n^{-3 / 4} u_{0} .
$$

Using our earlier techniques (as in the reduction of (5.2) using (5.3)), we see easily that, the probability limits being conditional on $K_{n}=k_{n}$,

$$
\begin{aligned}
& \operatorname{plim}_{n \rightarrow \infty} n^{3 / 4}\left[T_{n}\left(n^{-\frac{1}{2}}\left(s+k_{n}\right)-T_{n}\left(n^{-\frac{1}{2}} s, p\right)+n^{-3 / 4} u_{1}, p\right)\right. \\
& \left.\quad-T_{n}\left(n^{-\frac{1}{2}}\left(s+k_{n}\right), p\right)\right]=0, \\
& \operatorname{plim}_{n \rightarrow \infty} n^{3 / 4}\left[T_{n}\left(n^{-\frac{1}{2}} k_{n}+n^{-3 / 4} u_{0}, p\right)-T_{n}\left(n^{-\frac{1}{2}} k_{n}, p\right)\right]=0,
\end{aligned}
$$

uniformly for $b^{-1}<k_{n}<b$, for each $b>0$. Hence, the limiting conditional probability of the event $\left\{U_{n}(s)<u_{1}, U_{n}(0)<u_{0}\right\}$ is that of the inequalities

$$
\begin{aligned}
& -n^{3 / 4}\left[T_{n}\left(n^{-\frac{1}{2}}\left(s+k_{n}\right), p\right)-T_{n}\left(n^{-\frac{1}{2}} s, p\right)\right]<u_{1}, \\
& -n^{3 / 4} T_{n}\left(n^{-\frac{1}{2}} k_{n}, p\right)<u_{0},
\end{aligned}
$$

providing these last limiting probabilities are continuous in $u_{0}, u_{1}$, which will turn out to be the case. But the two random variables on the left sides of the inequalities (5.8) are, by the Central Limit Theorem, asymptotically conditionally jointly normal (uniformly in $b^{-1}<k_{n}<b$ ) with means 0 , variances $k_{n}$, and covariance $\max \left(k_{n}-s, 0\right)$. Thus, $U_{n}(0) / k_{n}^{\frac{1}{2}}$ and $U_{n}\left(k_{n} t_{2}\right) / k_{n}{ }^{\frac{1}{2}}$ have conditional limiting law equal to that described for $J(0)$ and $J\left(t_{2}\right)$ above (1.7). The general approach of finite-dimensional conditional laws of the $U_{n}$-process to the finitedimensional laws of the $J$-process is obtained similarly. 
Next, we show that, for each $c>0$,

$$
\begin{aligned}
\lim _{\epsilon \rightarrow 0} \varlimsup_{n \rightarrow \infty} \sup _{\left|p^{\prime}-p\right| \leqq \tau n^{-1 / 2 \epsilon}} \epsilon^{-1} P\left\{\sup _{|t|<\epsilon} n^{3 / 4} \mid R_{n}\left(p^{\prime}\right.\right. & \left.+n^{-\frac{1}{2}} t\right) \\
& \left.-R_{n}\left(p^{\prime}\right) \mid>c\right\}=0 .
\end{aligned}
$$

From this it follows that, writing $B_{\varepsilon}=\{j:|j| \leqq \tau / \epsilon, j=$ integer $\}$, by choosing $\epsilon$ sufficiently small one can make

$$
P\left\{\sup _{|t| \leqq \tau} \pm R_{n}\left(p+n^{-\frac{1}{2}} \tau\right)-\sup _{j \varepsilon B_{\epsilon}} \pm R_{n}\left(p+n^{-\frac{1}{2}} j \epsilon\right)>n^{-3 / 4} c\right\}
$$

as close to zero as desired for all large $n$. Since $B_{\epsilon}$ is finite, the proof that $\sup _{|t| \leqq \tau} U_{n}(t) \rightarrow \sup _{|t| \leqq \tau} J^{*}(t)$ in law follows at once from the convergence of finite-dimensional laws proved above, in the same manner that the analogous sample df deviation results follow from such convergence and the smallness of the analogue of (5.10) in [3] and [8]. Convergence in law of $f\left(U_{n}\right)$ to $f\left(J^{*}\right)$ for functionals $f$ on the space of functions on $[-\tau, \tau]$ continuous except for finitely many finite jumps, and such that $f$ is continuous in the uniform topology wp 1 according to the law of $J^{*}$, is then proved in the same way by approximating such functionals in the manner of Donsker [3], page 281, so that the result for the approximating functional can be obtained from the result for $\sup _{\tau \varepsilon L} \pm U_{n}(t)$ for various intervals $L$.

By (1.1), relation (5.9) will be proved if we prove each of the two statements obtained from it by replacing $R_{n}(x)$ by (i) $S_{n}(x)-x$ and by (ii) $Y_{x, n}-x$. For (i), we thus consider

$$
P\left\{T_{n}^{*}\left(\epsilon n^{-\frac{1}{2}}, p^{\prime}\right)>n^{-3 / 4} c\right\} .
$$

(The corresponding expression with $\epsilon$ replaced by $-\epsilon$ is treated similarly.) As $n \rightarrow \infty$, the expression $[M / a(1+w)]^{\frac{1}{2}} w(\delta-a)$ of $(2.7)$, with $\delta=\frac{1}{2}$, approaches $c / 2 \epsilon^{\frac{1}{2}}$. Hence for all $\epsilon<c^{2} / 20$ we can apply the first two lines of (2.10) and obtain, for all $n>N_{0}$ (where $N_{0}$ is independent of $\epsilon$ as long as $\epsilon<c^{2} / 20$ ), that the expression (5.11) is no greater than

$$
\begin{aligned}
\frac{4}{3} P\left\{\left|T_{n}\left(\epsilon n^{-\frac{1}{2}}, p^{\prime}\right)\right|>\right. & \left.n^{-3 / 4} c\right\} \\
& =\frac{4}{3}\left\{B^{*}\left(-n^{\frac{1}{4}} c-, n, \epsilon n^{-\frac{1}{2}}\right)+1-B^{*}\left(n^{\frac{1}{4}} c, n, \epsilon n^{-\frac{1}{2}}\right)\right\} \\
& \sim \frac{8}{3} \Phi\left(-c / \epsilon^{\frac{1}{2}}\right)
\end{aligned}
$$

uniformly for $p^{\prime}$ in any closed interval excluding 0 and 1 , as $n \rightarrow \infty$, this last by the Central Limit Theorem with error term. Since $\lim _{\epsilon \rightarrow 0} \epsilon^{-1} \Phi\left(-c / \epsilon^{\frac{1}{2}}\right)=0$, the result for (i) is complete.

As for (ii), consider first the expression

$$
P\left\{\sup _{0<t<\epsilon}\left|Y_{p^{\prime}+n^{-\frac{1}{3} t, n}}-Y_{p^{\prime}, n}\right|>n^{-3 / 4} c \mid Y_{p^{\prime}, n}=y, S_{n}(y)=p+n^{-1} \theta\right\},
$$

where $0 \leqq \theta<1$ accounts for excess of $S_{n}(y)$ over $p$ due to the jump at $y$. A moment's reflection shows that under the conditioning of (5.13), the event

$$
\left\{T_{n}^{*}\left(n^{-\frac{1}{2}} \epsilon+n^{-3 / 4} c, y\right)<n^{-3 / 4} c-n^{-1}\right\}
$$


entails the complement of the main event in (5.13). The event (5.14) is very similar to the complement of the event of (5.11), except that we must now compute the conditional probability under the condition of (5.13), for $y$ in a small neighborhood of $p^{\prime}$ of probability approaching 1 with $n$, e.g., for $\left|y-p^{\prime}\right|<n^{-\frac{1}{4}}$. One obtains without difficulty, for the complement of (5.14), an analogue of (5.12). One must then consider (5.13) with $-t$ replacing $t$, and there is no essential difference. Thus, (5.9) is established.

Finally we show that the $J^{*}$ sample paths are, wp 1, Lip $\alpha$ for all $\alpha<\frac{1}{2}$ by showing this for the paths of the $J$-process. From (1.7) we have $J(t+h)-J(t)$ distributed as $N(0,2|h|)$, so that for $m$ a positive integer we have

$$
E|X(t+h)-X(t)|^{2 m}=(2 m) !|h|^{m} / m !
$$

From Loève (1960), page 519, we conclude that the $J$-paths are, wp 1 , Lip $(m-1) / 2 m$ for every $m>0$. This completes the proof of Theorem 2 . See also Remark 5 of Section 6 .

6. Further results and remarks:

1. In view of the asymptotic normality of $U_{n} /\left|K_{n}\right|^{\frac{1}{2}}$ discussed below (1.5) (or that of the process $U\left(\left|K_{n}\right| t\right) /\left|K_{n}\right|^{\frac{1}{2}}$ discussed just above (1.7), or its more symmetric variant $\left.U\left(\left|K_{n}\left(p+n^{-\frac{1}{2}} t\right)\right| t\right) /\left|K_{n}\left(p+n^{-\frac{1}{2}} t\right)\right|^{\frac{1}{2}}\right)$, one may be led to inquire about the analogue of Theorem 1 or 3 for this process. The process resulting from this normalization of $U$ by division by the chance variable $\left|K_{n}\right|^{\frac{1}{2}}$ seems much less natural and interesting to the author than does Bahadur's $U$. Much of the technique of Section 3 and 4 can still be applied to the altered problem; the values of $K_{n}$ near 0 , and their oscillation with $n$, now cause extra difficulties.

2. Of much greater interest than the result of Theorem 3 would be the analogue for $\sup _{0<p<1} \pm R_{n}(p)$, for simplicity when the $X_{i}$ are uniform (or for $\sup _{-p<t<1-p} U_{n}\left(n^{\frac{1}{2}} t\right)$, to which one can apply an analogue of Lemma 1 if $\inf _{x \varepsilon J} F^{\prime}(x)>0$ on an interval $J$ for which $\left.P\left\{X_{1} \varepsilon J\right\}=1\right)$. Here the methods we have used herein do not even yield a weak law, analogous to $\sup _{|t| \leqq \tau}\left[ \pm U_{n}(t)\right]$ approaching in law $\sup _{|t| \leqq \tau}\left[ \pm J^{*}(t)\right]$. (In particular, if $\sup _{0<p<1} c_{n} R_{n}(p)=\mathrm{O}(1)$ in probability, then a separable process whose finite-dimensional distributions are the limiting ones for $c_{n} R_{n}(\cdot)$, is the process which has sample function identically 0 , wp 1.) Some bounds on the law of $\sup _{p} \pm R_{n}(p)$ have, however, been obtained. For example it is exactly of order $(\log n)^{\frac{1}{2}}$ in probability. We shall return to this topic in another paper.

Much simpler is the consideration of $R_{n}(p)$ or the $U_{n}$ processes corresponding to a fixed finite collection of values $p$, which are seen from Section 1 to be asymptotically independent. Moreover, Theorem 3 immediately yields its analogues for maxima of these quantities.

3. Bahadur has mentioned in [1] that the law of the iterated logarithm (L.I.L.) for sample quantiles follows at once from his estimate on $R_{n}$. In fact, much more is true: not only can one obtain in this way a classical form of the L.I.L. (Equation (4) of [1]), which can also be obtained directly but not as quickly by modifications of the standard proof for sums of Bernoulli random variables (as in [6]), 
but also by using Bahadur's elegant device one can obtain at once the much more difficult strong form [5] from that which delimits the upper and lower classes for increasing sequences $\left\{c_{n}\right\}$ of positive values in the Bernoulli case as

$$
\begin{aligned}
\sum_{n} n^{-1} c_{n} e^{-\frac{1}{2} c_{n}^{2}} & =\infty \Leftrightarrow 1=P\left\{ \pm n^{\frac{1}{2}}\left(S_{n}\left(\xi_{p}\right)-p\right)>\sigma_{p} c_{n} \text { i.o. }\right\} \\
& <\infty \Leftrightarrow 0=P\left\{ \pm n^{\frac{1}{2}}\left(\mathrm{~S}_{n}\left(\xi_{p}\right)-p\right)>\sigma_{p} c_{n} \text { i.o. }\right\}
\end{aligned}
$$

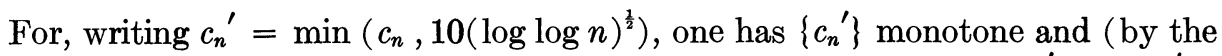
ordinary L.I.L. for $S_{n}\left(\xi_{p}\right)$ ) in the same class as $\left\{c_{n}\right\}$. By Feller [5], $c_{n}{ }^{\prime} \pm 1 / c_{n}{ }^{\prime}$ is in the same class as $\left\{c_{n}{ }^{\prime}\right\}$, as is therefore $\left\{c_{n}{ }^{\prime} \pm \frac{1}{10}(\log \log n)^{-\frac{1}{2}}\right\}$ and hence (again by the ordinary L.I.L. for $\left.S_{n}\left(\xi_{p}\right)\right)\left\{c_{n} \pm \frac{1}{10}(\log \log n)^{-\frac{1}{2}}\right\}$. But (1.2) or (1.3) yields $\left|n^{\frac{1}{2}} F^{\prime}\left(\xi_{p}\right) R_{n}(p)\right|<\frac{1}{10}(\log \log n)^{-\frac{1}{2}}$ for almost all $n$, wp 1 . Hence, we obtain

TheOREM 4. For $\left\{c_{n}\right\}$ positive and nondecreasing,

$$
\begin{aligned}
\sum_{n} n^{-1} c_{n} e^{-\frac{1}{2} c_{n}{ }^{2}} & =\infty \Leftrightarrow 1=P\left\{ \pm n^{\frac{1}{2}} F^{\prime}\left(\xi_{p}\right)\left(Y_{p, n}-\xi_{p}\right)>\sigma_{p} c_{n} \text { i.o. }\right\} \\
<\infty & \Leftrightarrow=P\left\{ \pm n^{\frac{1}{2}} F^{\prime}\left(\xi_{p}\right)\left(Y_{p, n}-\xi_{p}\right)>\sigma_{p} c_{n} \text { i.o. }\right\} .
\end{aligned}
$$

4. At present a strong form of Theorem 1, analogous to (6.2), is unknown. The methods used herein give reasonably sharp probabilistic bounds of the type usually required, but the approach of [5] for cumulative sums, especially in the case of the lower class, will require some delicacy to be carried over to the present problem.

5. The conclusion of Theorem 2 regarding $J^{*}$-paths being Lip $\alpha$ for all $\alpha<\frac{1}{2}$ wp 1 suggests that one should be able to proceed in the elegant manner of Lamperti [9] to the convergence of Theorem 2 by working in the Lip $\alpha$ space, replacing $S_{n}$ by the corresponding piecewise-linear continuous function $S_{n}{ }^{\prime}$ (say) so that the resulting $U_{n}$ is in $\operatorname{Lip} \alpha$. Unfortunately, this prescription cannot be carried out because Prokhorov's condition for the $U_{n}$ to lie, with high probability in a common compact set in the $\operatorname{Lip} \beta / \alpha$ space,

$$
E\left|U_{n}\left(p+n^{-\frac{1}{2}} t\right)-U_{n}(p)\right|^{\alpha} \leqq C|t|^{1+\beta}
$$

with $C, \alpha, \beta$ independent of $n$, cannot be verified. This is true even if one attempts only the classical sample df results (such as Kolmogorov-Smirnov) which consider $S_{n}$ rather than $U_{n}$ in this manner.

Incidentally, replacing $S_{n}$ by $S_{n}{ }^{\prime}$ changes $S_{n}$ by at most $1 / n$ at each point and also, with high probability, changes $Y_{p, n}$ by little, since the largest sample spacing in the uniform case is well-known to be of order $n^{-1} \log n$ wp 1. Thus, Theorems 1,2 , and 3 still hold if $S_{n}$ is replaced everywhere by $S_{n}{ }^{\prime}$, and one sees also the validity of our remark in Section 1 concerning the irrelevancy to our results of the manner of definition of $Y_{p, n}$ when there is ambiguity due to $n p$ being an integer.

6. It would be interesting to investigate the efficacy of statistical procedures based on $R_{n}(p)$, $\sup _{0<p<1} R_{n}(p)$, and other variants of Bahadur's statistic; the "cancellation" of much of the information in $Y_{p, n}$ and $S_{n}\left(\xi_{p}\right)$, and the resulting smaller order of $R_{n}(p)$, make one wonder whether there are many meaningful 
applications. As a trivial example, if $X_{1}, X_{2}, \cdots, X_{n}$ are independent and symmetrically distributed about 0 , one could use $\left(\frac{1}{2}-S_{n}(0)\right) / Y_{\frac{1}{2}, n}$ to estimate the probability density function at 0 , but this is not very efficient. See Section 3 of [1a] for Bahadur's use of $R_{n}(p)$ to reduce asymptotic consideration of certain nonlinear statistics to that of linear ones.

7. Let $\eta^{(1)}(n)=n\left(p-S_{n}\left(\xi_{p}\right)\right) / \sigma_{p}$ and $\eta^{(2)}(n)=n F^{\prime}\left(\xi_{p}\right)\left(Y_{p, n}-\xi_{p}\right) / \sigma_{p}$, and extend these to $\eta^{(i)}(t)$ for positive real $t$ by linear interpolation between successive integer arguments. Write $\eta_{n}{ }^{(i)}(t)=(2 \log \log n)^{-1 / 2} \eta^{(i)}(n t)$. The beautiful results of Strassen [11] include $\eta^{(1)}$ as a special case. Since our Theorem 1 or (1.2) implies that

$$
\lim _{T \rightarrow \infty}(2 T \log \log T)^{-1 / 2} \sup _{t \leqq T}\left|\eta^{(1)}(t)-\eta^{(2)}(t)\right|=0 \text { wp } 1,
$$

we obtain

Theorem 5. Strassen's Theorems 2 and 3 hold with $\eta^{(1)}($ his $\eta)$ replaced by $\eta^{(2)}$. Consequently, the results of Section 3 of [11] hold with $\eta^{(1)}(n)\left(\right.$ his $\left.S_{n}\right)$ replaced by $\eta^{(2)}(n)$.

The second part of Theorem 5 follows from the way in which Strassen's Theorems 2 and 3 and elementary integrability or moment considerations are used in the proofs of his Section 3. In particular, his paragraphs (i)-(vi), the Corollary to his Theorem 3, and his equations (3), (4), (11) are valid for $\eta^{(2)}(n)$, as are any other results corresponding to ones for $\eta^{(1)}(n)$ obtained from his Theorems 2 and 3 by such methods. Bahadur has informed the author that Bickel independently suggested that direct study of the $\eta^{(2)}(n)$ process might yield such results as the L.I.L. for $Y_{p, n}$. I have not considered here direct study of the $\eta^{(2)}$-process starting from scratch, since the indirect approach using the labors of Bahadur and Strassen yields our Theorem 5 so easily, and since results like those of [11] have not yielded the strong form (6.1)-(6.2) of the L.I.L. One can also obtain most of the analogues of Strassen's Section 3 by applying Bahadur's bound directly there without recourse to Theorems 2 and 3 of [11].

8. The author's attention has recently been called to an abstract of Eicker [4] concerning Bahadur's bound (1.2) and the fact that it cannot be improved using only the Borel-Cantelli Lemma as in [1] (to be contrasted with the present approach which yields (1.3) by using the $n_{r}$ ). Eicker states that he conditions on the value of $Y_{p, n}$ rather than on $K_{n}$ or $S_{n}\left(\xi_{p}\right)$ as herein, but it seems unlikely that one would achieve any shortening of the present proof from the use of that conditioning.

The author is indebted to Roger Farrell for helpful discussions.

\section{REFERENCES}

[1] Bahadur, R. R. (1966). "A note on quantiles in large samples". Ann. Math. Stat. 37 577-580.

[1a] BaHAdUR, R. R. (1967). "Rates of convergence of estimates and test statistics". Ann. Math. Stat. 38 303-324.

[2] Chung, K. L. (1949). "An estimate concerning the Kolmogoroff limit distribution". Trans. Amer. Math. Soc. 67 36-50. 
[3] Donsker, M. D. (1952). "Justification and extension of Doob's heuristic approach to the Kolmogorov-Smirnov Theorems". Ann. Math. Stat. 23 277-281.

[4] EICKER, F. (1966). "On the asymptotic representation of sample quantiles" (abstract). Ann. Math. Stat. 371425.

[5] FeLlek, W. (1943). "The general form of the so-called law of the iterated logarithm". Trans. Amer. Math. Soc. 54 373-402.

[6] FelLer, W. (1957). An Introduction to Probability Theory and its Applications. 1 (2nd edition). John Wiley \& Sons, N. Y.

[7] KIEFER, J. (1961). "On large deviations of the empiric d.f. of vector chance variables and a law of the iterated logarithm". Pac. Math. 11 649-660.

[8] KIEFER, J. and Wolfowitz, J. (1958). "On the deviations of the empiric distribution function of vector chance variables". Trans. Amer. Math. Soc. 87 173-186.

[9] Lamperti, J. (1962). "On convergence of stochastic processes". Trans. Amer. Math. Soc. 104 430-435.

[10] Lòve, M. (1960). Probability Theory (2nd edition). D. Van Nostrand Princeton.

[11] Strassen, V. (1964). "An invariance principle for the law of the iterated logarithm". Z. Wahrscheinlichkeisstheorie und Verw. Gebiete 3 211-226. 